\title{
Formação do Atendente de Enfermagem no Brasil: Um Desafio
}

\author{
Ana Luiza Stiebler Vieira*
}

Um levantamento realizado pelo Núcleo de Recursos Humanos da Ensp/Fiocruz, em 1984, mostrou que, em nosso país, no setor saúde, $61,3 \%$ da força de trabalho se distribuem entre médicos e atendentes de enfermagem. Tendo em vista os princípios da Reforma Sanitária, intentando configurar o Sistema Unico de Saúde com a perspectiva de avaliaçāo constante de sua resolutividade, percebe-se que uma das dificuldades consiste na escolaridade e formação do atendente de enfermagem. No Conselho Federal de Enfermagem (Registro e Cadastramento), até fevereiro de 1989 estão computados 98.770 auxiliares de enfermagem dos quais $10 \%$ com formação "supletiva" e $90 \%$ via formal.

Correlacionando-se a atual demanda de atendentes com a oferta de cursos de auxiliar de enfermagem de cada estado, chega-se à conclusāo de que o tempo exigido para esta formação é de 10 anos para $60 \%$ dos estados, 67 anos para Alagoas e de 3 anos para o Piauí.

Conclui a autora ser necessário, para suplementar esta formação, vontade política, co-participando ministérios, instituiçōes e entidades de categorias para abertura de cursos descentralizados, além de outras medidas (liberação de bolsas; licenças de horário parcial e rotativo de trabalho; remanejamento de horário de serviço etc), para torná-la acessivel a sua clientela.

\section{INTRODUÇÃO}

Os níveis médio e elementar participam no conjunto da força de trabalho em saúde, de forma intensa. Em 1984 , este contingente representou $60 \%$ dos empregos em saúde, sendo que, em décadas anteriores, o atendente tinha presença hegemônica. Com o crescimento verificado, entre 1976 a 1984 , dos auxiliares e técnicos de enfermagem de $11,6 \%$ e $10,3 \%$, respectivamente,

*Enfermeira com especialização em saúde páblica e epidemiologia. Escola Nacional de Saúde Pública. Departamento de Administração e Planejamento em Saude. Núcleo de Recursos Humanos em Saúde. houve desaceleração da expansão de empregos para os atendentes, representando estes $50 \%$ dos empregos, de níveis médio e elementar.

A pesar desta desaceleração, o contingente de atendentes de enfermagem, no Brasil, é grande (184.723), detendo $29,9 \%$ do total de postos de trabalho em Saúde, no Brasil, em 1984.

Cadernos de Saúde Pública, RJ, 6(1): 62-73, jan/mar, 1990. 
Diante desta imensa desqualificação profissional, da intencionalidade do Sistema Único de Saúde, de avaliação constan te de sua resolutividade, da resolução do Conselho Federal de Enfermagem de qualificar estes atendentes num prazo de dez anos, objetivamos:

- avaliar, quantitativamente, as vias de formação para - auxiliar de enfermagem até então utilizadas;

- configurar, através das recentes autorizaçōes concedidas para o nivel elementar, o quadro geral desta categoria, por estado, correlacionado-o com a oferta de cursos;

- detectar os óbices para a sua formação;

- sugerir gestōes a serem en frentadas para a consecução da qualificação do atendente.

\section{METODOLOGIA}

Considerando o crescimento de auxiliares de enfermagem, no período de 1976 a 1984 , de $11,6 \%$, fomos buscar, junto ao Conselho Federal de Enfermagem ( $\mathrm{Co}$ fen), nos 98.770 registros dos auxiliares (correspondentes de novembro de 1975 a dezembro de 1988 , contidos em 165 livros), os percentuais destes com formação via supletiva e via formal.

Foram coletados dados tais como: número de auxiliar de enfermagem (via supletiva) por ano, estado e idade ao se registrar no respectivo órgão. Foram colhidos, também, no Cofen, na Unidade de Registro e Cadastro, o número de autorizaçōes concedidas ao atendente de enfermagem desde a sua reabertura em a gosto de 1987 até $31.12 .88^{*}$ e o número de escolas de auxiliar e técnico de enfermagem, por cada estado.

Ressal tamos que o percentual de auxiliares de en fermagem, registrados com formação via supletiva, não corresponde à totalidade destes no país. O processo de registro não especifica se cursos particulares ou de in stituiçōes públicas são conveniados e/ ou autorizados para a formação via supletiva. Optamos, então, por assinalar os cursos explicitamente declarados supletivos, totalizando 29 para todos os estados.

Para se ter controle da situação, seria necessário verificar em cada Secretaria Estadual de Educação este percentual, demandando um estudo mais amplo e custoso. Tal fonte nos daria o total de formados, provavelmente maior do que o número de auxiliares de enfermagem cadastrados no Cofen, já que o órgão não detém $100 \%$ do registro dos profissionais.

Se todos os 29 cursos se mantivessem em atividade, em 1987, representariam $9,2 \%$ e, em $1988,8,6 \%$ do total (339) de cursos de auxilares de enfermagem existentes no Brasil. Tal fato evidencia a complexidade deste tipo de estudo para se tentar controlar todos os cursos e centros de exames no país (conveniados
*Ean 1978, foram suspensas as autorizaçóes do nível elementar de enfermagem, de acordo com - despacho do Ministério do Trabalho publicado no D.O.U. de 18.10 .78 , fls. 18.817 , e reabertas conforme Resoluçáo Cofen $n: 91$ e n: 96 de 1987. 
ou autorizados); considerando a variação numérica anual, data de abertura e fechamento dos cursos, e dupla modalidade de formação (regular e supletivo) numa mesma instituição.

\section{RESULTADOS}

O registro no Cofen corresponde à inscrição definitiva no órgão. Inclui pessoas que já não estão mais em atividade, e exclui temporariamente, os profissionais recém-inscritos, portadores de franquia provisória para o exercício profissic .1. Iniciando o Cofen o registro em novembro de 1976 , este nos fornece a população de auxiliares via supletiva (com as ressalvas anteriores), desde o início, com a lei 5692, de 1971 .

A té 31 de dezembro de 1988 , foram registrados 98.770 auxiliares de enfermagem, com taxa de crescimento anual de $28,0 \% ; 18,2 \% ; 16,7 \% ; 20,1 \% ; 17,8 \%$; $16,2 \% ; 12,8 \% ; 10,5 \% ; 11,6 \% ; 10,2 \% ; 10,5 \%$; $11,2 \%$; e $1,3 \%$ de 1976 a 1988 . As taxas mais elevadas, nos primeiros anos, demostra um in tenso registro de profissionais já qualificados em períodos anteriores a 1976.

Entre este total dos registros de auxiliares de enfermagem, foram encontrados 9.778 com formação via supletiva, o que corresponde a $9,9 \%$ do total de dezembro de 1988 , demonstrado na tabela $\mathrm{I}$.

1978 , ano em que começam a aparecer os registros de formação via supletiva, demonstra o maior percentual, considerado resultante da Lei 5692, de 1971. A suspensão da autorizaçāo de atendentes, neste mesmo ano, foi, talvez, o fato que contribuiu para novamente elevar o percentual de formação supletiva a partir de 1981 , mantendo-se até 1986 com uma média anual de 10,9\%. Em 1987 e 1988 os percentuais se mostram bastante inferiores, fato talvez decorrente da reabertura das autorizaçōes, ou mesmo falta de tempo hábil para conclusão da formação auxiliar para os recém-autorizados. Provavelmente a partir de 1989 poderemos verificar maiores percentuais.

$\mathrm{Na}$ tabela II, podemos verificar a distribuição dos auxiliares de enfermagem com formação via supletiva por estados e regiōes brasileiras.

Nota-se que a região Sudeste teve o melhor desempenho, com percentual de $45,6 \%$, basicamente sustentado pelos estados do Rio de Janeiro e São Paulo. A regiäo Sul teve "maior desempenho, tendo inclusive os estados do iaraná e Rio Grande do Sul como também o de Minas Gerais apresentado percentuais inferiores ao de Roraima. Em ordem decrescente, os estados do Rio de J aneiro, Goiás, São Paulo, Roraima, Pemambuco e Rio Grande do Norte tiveram melhor desempenho na formação via supletiva. 
Tabela I Númer o e Percentual de Auxilares de Engermagem com Formação Via Supletiva de 1978 a 1988

\begin{tabular}{|c|c|c|}
\hline \multirow{2}{*}{ Ano } & \multicolumn{2}{|c|}{ Aux. Enf. (Via Supletivo) } \\
\hline & $\mathrm{N}:$ & $\%$ \\
\hline 1978 & 1507 & 15,4 \\
\hline 1979 & 557 & 5,7 \\
\hline 1980 & 489 & 5,0 \\
\hline 1981 & 919 & 9,4 \\
\hline 1882 & 1271 & 13,0 \\
\hline 1983 & 997 & 10,2 \\
\hline 1984 & 1164 & 11,9 \\
\hline 1985 & 1124 & 11,5 \\
\hline 1986 & 909 & 9,1 \\
\hline 1987 & 548 & 5,6 \\
\hline 1988 & 293 & 3,0 \\
\hline Total & 9778 & 100 \\
\hline
\end{tabular}

Fonte: Livios de Registro do Cofen, Unidade de Registro e Cadastro.

Dos 9.778 auxilianes de en fermagem com fomaçāo via supletiva $70,9 \%$ tinham até 39 anos de idade na data do registro no Cofen.

Cadernos de Saúde Pública, RJ, 611): 62-7; jan/mar, 1990. 
T abela II - Número e Percentual de Aux. Enf. (Supletivo) Registrados no Cofen de novembro de 1975 a 31.12.88, por estados e regiỏes do Brasil

\begin{tabular}{|c|c|c|c|c|c|}
\hline Estado & $\mathrm{n}:$ & $\%$ & Regiōes & n: & $\%$ \\
\hline$A M$ & 108 & 1,1 & Norte & 1114 & 11,7 \\
\hline RO & 616 & 6,3 & & & \\
\hline $\mathbf{R R}$ & 88 & 0,9 & & & \\
\hline PA & 332 & 3,4 & & & \\
\hline CE & 293 & 3,0 & Nordeste & 1730 & 17,7 \\
\hline PI & 59 & 0.6 & & & \\
\hline PB & 224 & 2.3 & & & \\
\hline $\mathrm{PE}$ & 577 & 5,9 & & & \\
\hline SE & 10 & 0.1 & & & \\
\hline $\mathbf{B A}$ & 59 & 0,6 & & & \\
\hline $\mathbf{R N}$ & 508 & 5,2 & & & \\
\hline MG & 68 & 0,7 & Sudeste & 4458 & 45,6 \\
\hline RJ & 2826 & 28,9 & & & \\
\hline$S P$ & 1437 & 14,7 & & & \\
\hline ES & 127 & 1,3 & & & \\
\hline MT & 342 & 3,5 & $\begin{array}{l}\text { Centro- } \\
\text { Oeste }\end{array}$ & 2191 & 22,4 \\
\hline $\mathrm{DF}$ & 49 & 0,5 & & & \\
\hline GO & 1800 & 18,4 & & & \\
\hline PR & 20 & 0,2 & Sul & 255 & 2,6 \\
\hline RS & 49 & 0,5 & & & \\
\hline SC & 186 & 1,9 & & & \\
\hline Total & 9778 & 100,0 & & 9778 & 100,0 \\
\hline
\end{tabular}

Fonte: Livros de Registro do Cofen, Unidade de Registro e Cadastro. 
Tabela III - Número do Pessoal de Nível Elementar Autorizado pelos Cofens até 31.12.88 e Número de Cursos de Auxiliar e Técnico de Enfermagem disponíveis em cada estado, em 1988.

\begin{tabular}{|c|c|c|c|}
\hline Estad $\propto s$ & $\begin{array}{l}\text { A tendentes } \\
\text { e outros }\end{array}$ & $\begin{array}{l}\text { Cursos Aux. } \\
\text { Enfermagem }\end{array}$ & $\begin{array}{l}\text { Cursos téc. } \\
\text { Enfermagem }\end{array}$ \\
\hline $\mathrm{AL}$ & 2.005 & 1 & 1 \\
\hline $\mathrm{AM}$ & 149 & 1 & \\
\hline BA & 2.350 & 19 & 7 \\
\hline $\mathrm{CE}$ & 575 & 5 & 3 \\
\hline $\mathrm{DF}$ & 1.221 & 2 & 1 \\
\hline ES & 948 & 5 & 3 \\
\hline GO & 760 & 8 & 9 . \\
\hline MA & 700 & 9 & 12 \\
\hline MG & 10.988 & 31 & 41 \\
\hline PA & 3.500 & 4 & 1 \\
\hline$P E$ & 1.689 & 16 & 11 \\
\hline PB & 1.147 & 3 & 6 \\
\hline PI & 144 & 2 & 3 \\
\hline PR & 8.000 & 13 & 3 \\
\hline $\mathrm{RJ}$ & 2.968 & 63 & 90 \\
\hline RN & 1.100 & 5 & 3 \\
\hline RS & 10.451 & 28 & 15 \\
\hline SE & 351 & 4 & 4 \\
\hline$S C$ & 2.084 & 17 & 11 \\
\hline SP & 35.100 & 92 & 80 \\
\hline $\mathrm{MT}$ & 533 & 10 & 5 \\
\hline MS & 44 & 1 & \\
\hline TOTAL & 86.807 & 339 & 381 \\
\hline
\end{tabular}

Fonte: Cofen, Unidade de Registro e Cadastro. 
*Lei n: 7498, de 25 de jumho de 1986, regulamentada pelo decreto $\mathrm{n}$ : 94.406 , de 8 de junho de 1987.
Com as Resoluçōes 91 e 96, do Cofen, em 1987 , baseadas na nova legislação do exercício da enfermagem*, especificamente no art. 23 , que confere competência ao Cofen para autorizar o exercício profissional do nivel elementar, por dez anos, condicionado ao compromisso de formação profissional destes, num prazo mínimo de dois anos; o Conselho Federal de Enfermagem reabriu as autorizações neste ano. De 1987 até 31 de dezembro de 1988 , foram concedidas 86.807 autorizaçōes pelos Corens em todo o país. Esta distribuição por estado e o número de escolas disponíveis de auxiliar e técnico de enfermagem podem ser notados na tabela III.

Acreditamos que o total de autorizados não corresponde à totalidade do pessoal de nível elementar existente no pais e que nem o prazo máximo concedido para as autorizações ( 2 de janeiro de 1989) cobriu este contingente. E de se esperar conforme as cláusulas da Resolução 91, que tenham se apresentado pessoas que já tinham ou estão cursando o 1: grau, ou curso de auxiliar, técnico ou supletivo. A tabela III e o cadastramento do pessoal de enfermagem no Brasil até 5 de maio de $1988^{*}$, que mostram a concentração destes na regiāo Sudeste $(62,0 \%)$, principalmente no Rio de J aneiro e São Paulo, denunciam o baixo percentual de autorização no Rio de Janeiro $(3,4 \%)$, sendo inclusive menor do que no Pará $(4,0 \%)$. Este estado e os demais da região Norte situam-se em último lugar na disponibilidade de recursos humanos em enfermagem, com $4,0 \%$ para toda a regiảo.

O próprio Cofen, em decisão informada na publicação "Normas e Notícias", de abril de 1989, reconhece o fato de nāo ter coberto todos os atendentes, reabrindo as autorizações a partir daquele mês, sem estipular, ainda, a data de encerramento.

\section{DISCUSSÃO}

O percentual encontrado de auxiliares com formação via supletiva $(9,9 \%)$, conforme consi deração metodológica anteriormente apontada, fica subestimado. Acrescenta-se, ainda, o fato de que nem todos que se formaram via supletiva, trabalhando ou não, estão registrados. Podemos sugerir que o resultado, no minimo, poderia ser duplicado, considerando-se que ape$\mathrm{n}$ as $10 \%$ da rede de cursos, particulares ou institucionais, ofertassem a via supletiva, seja por autorização ou convênio.

Apesar de apenas trabalharmos com 29 cursos, o desempenho. não foi insignificante. Entre o total de auxiliares de enfermagem registrados (de todo o tipo de formação), a via supletiva representou um percen-

Cadernos de Saúde Pública, RJ, 6(1): 62-73, jan/mar, 1990. 
tual máximo de 36,4\% (1978); mínimo de 2,9\% (1988) e médio de $14,1 \%$.

A idade predominantemente jovem $(70,9 \%$ até 39 anos) daqueles que procuram a formação via supletiva, considerando que esta força de trabalho é essencialmente constituída por mulheres (2), corresponde a percentuais encontrados por Machado (2), em 1980, em que $71,1 \%$ de mão-de-obra do pessoal de enfermagem tinham até 39 anos. Encontramos, na faixa de 40 a 59 anos, $28,6 \%$ e de 60 anos e mais, $0,5 \%$, equivalentes aos encontrados por esta mesma autora nas respectivas faixas de idade: $25 \%$ e $0,5 \%$.

Outra modalidade de formação mais recente é o Larga-Escala $(5,7)$, que é um programa de formação de pessoal, a nível médio e elementar, para os serviços básicos de saúde, com metodologia centrada na mudança comportamental do alunado, através da construção progressiva na sua prática ativa. Por ser recente e de processo lento, o programa não oferece impacto na formação do nível elementar.

Utilizando-se os dados da tabela III, sabendo-se a priori que o número de autorizados está subestimado, principalmente se considerarmos o levantamento feito por Santos (6), em 1984, no estado do Rio de Janeiro, que encontrou 4.693 atendentes, podemos comparar, por estado, qual seria o tempo necessário para as escolas de auxiliar de enfermagem formarem os atendentes, pressupondo que estas permaneçam em funcionamento; que todos os autorizados tenham a escolaridade de $1^{\circ}$. grau completo, e que cada curso forme trinta pessoas por ano.

Assim, otimizando os resultados, já que estamos superestìmando a escolarizaçāo e subestimando o número de atendentes, principalmente, se considerarmos as reaberturas das autorizaçóes, encontramos que apenas três estačos - Rio de Janeiro, Mato Grosso e Mato Grosso do Sul - encontram-se em situação mais favorável para formação do seu quadro elementar autorizado, no prazo de dois anos.

Os atendentes de todos os outros estados, obrigatoriamente, teriam sua autorização renovável, pois a demanda por cursos excede, em muito, a oferta. $O$ estado de Alagoas só conseguiria formar sua clientela em 67 anos; o Pará, em 29 anos: Paraná, em 20,5 anos; Distrito Federal, em 20,4 anos; Paraíba e São Paulo, em 12,7 anos; Rio Grande do Sul, em 12,4 anos; Minas Gerais, em 10,8 anos; Rio Grande do Norte, em 7,3 anos;Espírito Santo, em 6,3 anos; Amazonas, em 5 anos; Bahia, em 4,5 anos; Ceará e Santa Catarina, em 4 anos; Pernambuco, em 3,5 anos; Goiás, em 3,2 anos; Sergipe, em 2,9 anos. De acordo com as mesmas condições, o período requerido para as regiōes 
completarem esta formação é 67 anos para o Nordeste, 29 anos para o Norte e de 21 anos para o Centro-Oeste e o Sul.

Verificamos, pois, que a atual situação da formação do nível elementar, no Brasil, não poderá corresponder às expectativas do Cofen, que prevê um periodo máxinuo ue iú anos para o fornecimento de renovação de autorizaçãn. Prndurindo as escolas a nível otimizado, portanto não real, $40 \%$ dos estados necessitariam de um período mínimo de 10,8 anos e máximo de 67 anos. Pelos resultados, $60 \%$ dos estados poderiam formar em menos de 10 anos, necessitando em média de 4,2 anos, porém nāo se levou em consideração a dispersão das escolas e clientela e os outros fatores já citados, os quais obviamente impediriam esta tarefa dentro do prazo estipulado.

Tomando-se os dados de uma pesquisa do Cofen, em 1983 (1), sobre a escolarização de atendentes, apenas $22,8 \%$ destes tinham o 1 . grau completo, cursando o 1 . grau, $5,1 \%$ e incompleto, $36,2 \%$. Para o 2 . grau, o percentual de atendentes com curso completo foi de 15,2 , cursando, $3,9 \%$ e incompleto, $12,1 \%$.

A plicando os percentuais de escolarização completa, e cursando o $1^{\circ}$ e e $2^{\circ}$. graus $(47,0 \%)$ da pesquisa do Cofen, aos atuais atendentes autorizados (86.807), estes representam $44,7 \%$ do total da força de trabalho do atendente. Tal fato corrobora com a hipótese aventada anteriomente de que as pessoas que procuraram obter as autorizaçōes seriam aquelas que já estivessem em melhores condiçöes de escolaridade, e que o Cofen năo conseguiu autorizar a totalidade do nível elemen$\operatorname{tar}$.

Se aplicarmos os percentuais da pesquisa do Cofen dos atendentes com escolarização completa de 1: grau $(22,8 \%)$ e de $2^{\circ}$ grau $(15,2 \%)$ nos 86.807 autorizados, o número de anos necessário para a formação auxiliar, obviamente, diminui. Assim considerando, 13.195 pessoas do nível elementar poderiam utilizar os cursos de técnico de enfermagem ( 381 em todo o país, com exceção de Amazonas e Ma to Grosso do Sul), restando 73.612 atendentes para 339 cursos de auxiliar de enfermagem. Assim, em todo o país, no prazo de 10 anos, daria para formar 101.700 auxiliares. Obviamente tal estimativa não é real, pois, não consideramos por falta de dados, a distribuição dos atendentes por escolaridade em cada estado.

Embora a participação do atendente na equipe de saúde tenha diminuído entre 1976 a 1984 de $35,8 \%$ para $29,9 \%$, neste último ano ele ainda fazia a bipolarização com a mão-de-obra médica,cujo percentual de participação era de $31,4 \%$ (3). Antes de 1982 , o setor 
privado absorvia a maioria do nível elementar; o setor público a partir de meados de 1980 passa a absorvê-los mais, tendo seu contingente praticamente duplicado em oito anos: em 1976, com 45.558 , e,em 1984, com 85.517 postos de trabalho (3). Em 1984 ocupavam 184.723 empregos em saúde, sendo que $76,4 \%$ desempenhavam suas atividades em estabelecimentos hospitalares, no cuidado direto com o paciente. (3)

Entrevê-se, assim, que a "equipe de saúde" na verdade não existe, a clientela está sob os cuidados de profissionais especializados e de não-profissionais. Em $1980,76,0 \%$ destes últimos detinham rendimentos inferiores a três salários mínimos, o que provavelmente concorre para a rotatividade desta mão-de-obra, aliado ao fato de ser predominantemente jovem e constituída de mulheres. (2)

Baseados nestes fatos é que endossamos a resolução do Cofen, com a perspectiva de se melhorarem as chances daqueles que procuram os serviços de saúde, minimizando as suas iatrogenias, através da formação técnica deste contingente não-profissional. Perpetuar a nomeação de atendentes pela simples troca do instrumento de trabalho (da vassoura para a seringa, e da cor do uniforme) é não contribuir com a resolutividade e qualidade requeridas para a rede de saúde, tendo-se em vista os princípios da Reforma Sanitária.

Evidentemente, apresentamos um apanhado geral da situação do nível elementar no Brasil. Achamos que para a consecução da sua formação, conforme objetivos do Cofen, em cada estado deveriam ser levantados a escolaridade de seus autorizados, sua distribuição geográfica e institucional, o número e distribuição espacial das escolas formadoras, para, a partir daí, planejar a estratégia de capacitação.

Entendemos, também, que é somente através da vontade política e da intensa articulação do Cofen e dos Corens com entidades de classe, instituições de saúde e de educação, além de outras medidas (liberação de bolsas, licenças de horário parcial e rotativo de trabalho, remanejamento de horário de serviço, criação de cursos, convênios etc), que tomarão implementável a formação de nivel elementar, tomando-a acessível à sua clientela. Posteriormente à formação, que visa a melhoria do desempenho técnico baseado em conhecimento científico, as reciclagens e os treinamentos em serviço tornam-se indispensáveis.

Obviamente, as estratégias de ação e a escolha da via de formação estão diretamente ligadas às realidades locais.

Necessário torna-se, portanto, contribuir para que o paciente tenha invertido seu conceito adjetivo (resig- 
nado, sofredor, pessoa que padece ou vai padecer) para o gramatical: o que recebe a ação de agentes, mas de agentes terapêtuticos e nảo desencadeadores de iatrogenias.

Considering the intention of the Unified Health System in undertaking a permanent evaluation of its resolvability and the decision of the Federal Council of Nurses (FCN) in qualifying non-skilled assistants within a 10-year period, this study sought to determine the quantitative obstacles for this professional qualification. We investigated the means for assistant nurses formation using data derived from all assistant nurses registered at FCN, up to December 1988. We also collected the total number of non-skilled nursing-assistants with permission to practice and the number of courses available to assistant nurses and technicians in each Brazilian state. We concluded that in order to overcome the professional disqualification of this health labor force, it is necessary both political will and integrated participation of Ministries and Institutions, among other measures. The time required for achieving this objective based on the current courses offers is 10 years in $60 \%$ of the Brazilian states, where the maximum and minimum limits correspond to 67 years in the state of Alagoas and 3 years in the state of Piaui.

\section{REFERÈNCIAS BIBLIOGRÁFICAS}

1 - BRASIL. Conselho Federal de Enfermagem. $O$ exercicio da Enfermagem nas Instituiçóes de Saude do Brasil: 1982/1983. Conselho Federal de Enfermagem. Associaçáo Brasileira de Enfermagem. Rio de Janeiro. 1985. 1: volume.

2 - MACHADO, M.H. "A Participação da Mulher no Setor Saúde no Brasil - 1970/80". Cadernos de Saúde Pública, M.S. ENSP/FIOCRUZ. Rio de Janeiro, vol. 2, n: 4. Outubro/Dezembro de 1986.

3 - MINISTÉRIO DA SAÚdE. Fiocruz. Escola Nacional de Saúde Pública. Núcleo de Recursos Humanos em Saúde. Dinâmica do Emprego em Saúde no Brasil, 1976-1984. Rio de Janeiro, 1987. (mimeo).

4 - MÉDICI, A.C. "Emprego em Saúde na Conjuntura Recente: Lição para a Reforma Sanitária". Cadernos de Saúde Pública, M.S. ENSP/FIOCRUZ. Rio de Janeiro, vol. 2, n: 4. Outubro/Dezembro de 1986. 
5 - Programa de Formą̧ão em Larga Escala de Pessoal de Níveis Médio e Elementar para os Serviços Básicos de Saúde. Sintese da 1: Reunião de Acompanhamento do Projeto Larga Escala. Brasília. 8 a 9 de setembro de 1983.

6 - SANTOS, B.B. Atividades na Área de Formaçāo e Desenvolvimento de Recursos Humanos da Secretaria Estadual de Saúde do Rio de Janeiro. Trabalho de conclusão do Curso de Atualização em Desenvolvimento de Recursos Humanos da Escoli: Nacional de Saúde Pública. Rio de Janeiro. 1988 (mimeo).

7 - SANTOS, I. et. alli. Preparação de Pessoal pelas Instituiçōes de Saúde. Brasília. 1987. (mimeo). 